\title{
From Radiosonde to Papersonde: The Use of Conductive Inkjet Printing in the Massive Atmospheric Volume Instrumentation System (MAVIS) Project
}

\author{
Philip H. King ${ }^{1}$, James P. Scanlan ${ }^{2}$ and András Sóbester ${ }^{3}$ \\ University of Southampton, Southampton, SO16 7QF
}

\begin{abstract}
A promising method for the collection of atmospheric data is the en masse release of 'flocks' of nano Unmanned Aerial Vehicles (UAVs) from meteorological balloons. These UAVs would need to be lightweight, cheap, and disposable. Recent advances in Conductive Inkjet Printing (CIP) have allowed the fabrication of electronic circuits onto thin substrates, such as paper. We demonstrate that the direct printing of circuit tracks onto photo paper will allow the creation of such aircraft, which are not only disposable, but biodegradable. We present a prototype 'Papersonde', which features a directly printed circuit with a microprocessor, transceiver, barometer and temperature sensor. We also describe in detail the fabrication steps required to create the Papersonde. It is hoped that this circuit will form the basis of the nano UAVs we create for the Massive Atmospheric Volume Instrumentation System (MAVIS) project.
\end{abstract}

\section{Introduction}

$\mathrm{T}$ he use of unmanned aerial vehicles (UAVs) as sensor platforms promises to revolutionise the collection of environmental data. Small aircraft, the size of conventional remote-controlled planes, can, with the right combination of sensors and computational power, be programmed to survey the skies without constant operator intervention, greatly reducing operating costs. Current civilian applications of this technology include agricultural monitoring, ${ }^{1}$ atmospheric data gather in the lower atmosphere, ${ }^{2}$ and pollution monitoring. ${ }^{3}$

The current 'gold standard' for the collection of meteorological data is the balloon-launched radiosonde. These disposable sensor platforms are released every 12 hours from over 800 locations across the globe, and collect temperature, pressure and humidity data for use in weather prediction models. The position of the balloon as it ascends is normally determined using a built-in GPS chip, and data is transmitted to the ground as it is collected. Once the balloon has reached its maximum altitude, typically at between 7 and $35 \mathrm{~km}$ above sea level, it bursts, at which point the radiosonde is shut down and descends to the ground by parachute.

Traditional radiosondes have a number of drawbacks. Although by their design they are disposable, they are large and reasonably expensive. In addition, a single launch by definition only collects data along a single line through the atmosphere.

The Massive Atmospheric Volume Instrumentation System (MAVIS) project aims to build upon traditional radiosonde systems via the use of 'nano-scale', disposable UAVs. As outlined above, most UAVs used as sensor platforms for data gathering are the size of conventional remote control aircraft. They are built for use over multiple missions, use powered flight, and are built and controlled using methods and techniques normally used in larger manned aircraft. As a result, such UAVs are normally too large and heavy for launch via balloon (and thus have very limited altitude capabilities, and are too expensive to be considered disposable.

\footnotetext{
${ }^{1}$ Research Fellow, Faculty of Engineering and the Environment, Engineering Centre of Excellence, PMAIAA.

${ }^{2}$ Professor of Design, Faculty of Engineering and the Environment, Engineering Centre of Excellence, SMAIAA.

${ }^{3}$ Associate Professor, Faculty of Engineering and the Environment, Engineering Centre of Excellence, SMAIAA.
} 
Various groups have investigated the potential of ultralight 'nano' UAVs. For example, the Pounds research group at the University of Queensland have demonstrated prototype 'electronic paper planes' constructed from flex circuitry, for use in atmospheric data gathering and forest fire monitoring. ${ }^{4}$ However, although these aircraft are presumably affordable for disposable use, their fabrication using flex circuitry means that the release of large numbers of these aircraft would be ecologically unfriendly.

To build the MAVIS fleet, we have been exploring the potential of conductive inkjet printing (CIP) for the production of electronic circuits directly on paper substrates. This not only reduces the substrate cost, but also provides better compatibility with our goal of disposability, as the paper substrate is biodegradable. We have found that CIP allows the rapid prototyping and manufacture of complex circuits, with low setup costs. In this paper we will demonstrate our in-house development of CIP technology, and the production of a cheap, disposable 'Papersonde' that will form the basis of future MAVIS micro UAVs.

\section{Technology}

CIP technology has been used for a number of years, and usually involves the controlled deposition of inks containing silver nanoparticles. However, most of the techniques required secondary process steps in order to produce conductive circuits, such as high-temperature $\left(>150^{\circ} \mathrm{C}\right)$ sintering..$^{5}$ Although printing onto paper for RF applications using high temperatures has been demonstrated, ${ }^{6}$ we have found heating to such high temperatures often damages the paper, causing the sheet to warp.

However, recent breakthroughs have resulted in the production of "self-sintering" inks, that are compatible with photo papers. ${ }^{7}$ These inks have been previously demonstrated as suitable for the production of simple circuits. ${ }^{8}$ However, the use of CIP on paper for the production of the sort of complex circuits required for microcontrollers has yet to be achieved.

Self-sintering conductive inks are available from Methode Electronics Inc. (USA) and Mitsubishi Paper Mill (Germany). We have opted to use the Mitsubishi product, which we were able to acquire at a significantly lower cost than the Methode equivalent. We use a Brother MFC-J4410DW multifunction inkjet printer (Brother Industries,

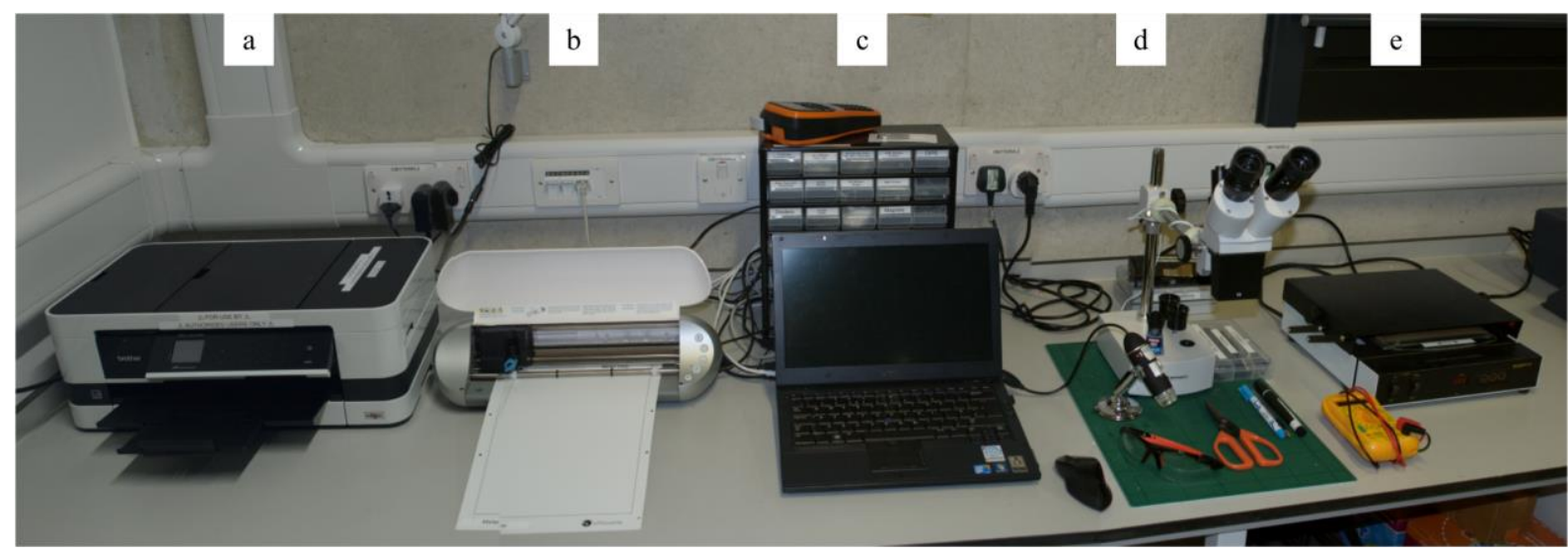

Figure 1. Conductive Inkjet Printing (CIP) production line. An overview of the equipment required for producing CIP circuits. An inkjet printer (MFC-J4410DW, Brother Industries Ltd., Japan) is used to print the circuits on photo paper (a). The printed substrate is then fed into a craft paper cutter, which is used to define the shape of the circuit, as well as allowing the creation of via holes in the top layer of the circuit. Mylar solder stencils can also be cut using this machine (b). The circuit is assembled under a stereozoom microscope (5802520 Stereomicroscope, Bresser, RS Components, UK) (d). Finally, the conductive epoxy, used as both adhesive and low-temperature solder, is cured at $70^{\circ} \mathrm{C}$ on a hotplate (Quick 450-870ESD, Kaisertech, UK) (e). Eagle CAD is used to create and export the circuit to Corel Draw, and integrated cutter software allows precise alignment between the printer's output and the cutter path (c). The total cost of this equipment (excluding laptop), including self-sintering conductive ink (NBSIJ-MU01, Mitsubishi Paper Mill, Düsseldorf, Germany) for $£ 220(100 \mathrm{ml})$ was around $£ 950$ including $20 \%$ UK VAT.

American Institute of Aeronautics and Astronautics 
Japan) to dispense the ink, along with non-OEM (Original Equipment Manufacturer) refillable cartridges.

A number of different cartridge combinations were tested, to test the feasibility of printing with both conductive and non-conductive inks. The hope was that the relatively expensive conductive ink could be saved by using standard ink for labelling, alignment marks, and logos for identification. However, the Brother inkjet print system nearly always mixes colour inks into the black during printing, even when instructed to print in black and white. This results in an increase in the track resistance of around 4 to 10 times compared to black ink alone (it is for this reason that clean non-OEM cartridges must be used, to eliminate standard ink contamination). Furthermore, as noted by Kawahara et al, ${ }^{8}$ by loading the conductive ink into all the available cartridge slots, the addition of 'coloured' inks allows the maximum volume of ink to be deposited, reducing track resistance. The resistance of the printed tracks is still greater by a factor of 8 to 10 than that of copper PCB tracks, but this is low enough to allow digital communication and the creation of complex CIP

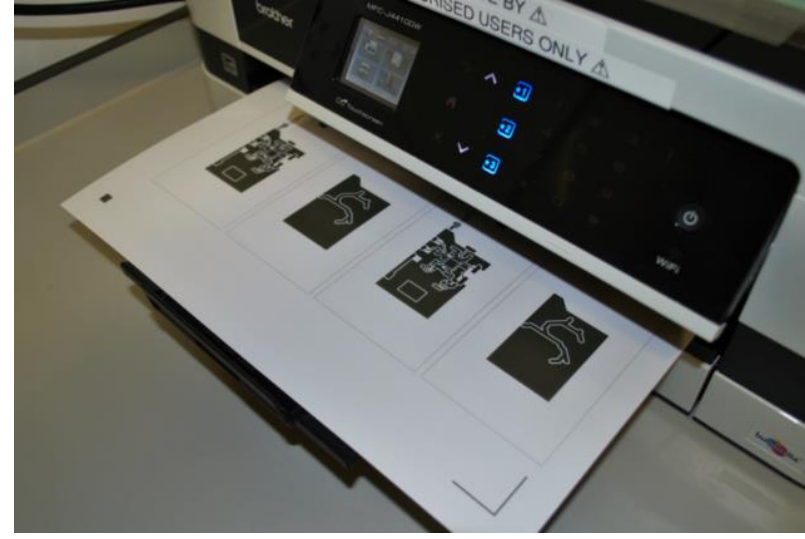

Figure 2. CIP output of consumer inkjet printer. Mutiple CIP circuits and circuit layers can be printed simultaneously, along with other features such alignment marks for use with the craft cutter (top and bottom left of the page). The ink is dry enough for further processing upon leaving the printer, although the printed circuit does not reach full conductivity for several hours. electronics.

As a substrate, we use Office Depot own-brand 'Glacier' 260 gsm Premium photo paper, as it allows excellent conductivity, is thick enough to be structurally useful in future micro UAVs, and is available for a reasonable cost ( $\$ 3.10$ for 20 sheets incl. $20 \%$ VAT). Normal office photocopy paper does not allow conductive tracks, as without the photo paper's coating the ink is absorbed into the paper, preventing contact between the suspended silver nanoparticles during the ink drying process.

The printing process is very simple. The PCB designs are created in Eagle CAD (CADSoft, USA) before being exported as a pdf into Corel Draw (Corel Corporation, Canada). The CIP PCB production itself is as simple as printing a document, as shown in Fig. 2. The resulting paper circuit requires drying before it reaches peak conductivity, but is touch-dry and ready for further processing immediately on exiting the printer.

Traditional prototype PCBs, such as strip board or breadboards, use through-hole components. However, the thickness of the paper for CIP circuits is insufficient to provide the mechanical anchoring required for these components to be useable. Therefore surface mount devices (SMDs) are used instead. SMD components are designed to be used in high-throughput electronic device fabrication, using automated pick-and-place robots, and are often very small in production electronics. However, larger sizes of passive components (resistors, capacitors, inductors etc.) are available, and 1206 and 0805 components are easily manipulated by hand, using tweezers. In order to ease this process, we have purchase a stereozoom microscope (58-02520 Stereomicroscope, Bresser, RS Components, UK).

When used with conventional PCBs or flex circuitry, SMD components are normally mechanically and electrically attached to the boards using solder paste. We have investigated a low temperature solder paste (Chip Quick SMDLTFP Solder Paste, Onecall, UK) for compatibility with CIP circuits on photo paper. However, as could be expected, it was found that heating the solder up to the required 'low temperature' of $140^{\circ} \mathrm{C}$ resulted in heavy warping of the paper substrate, and the photo coating separating from the bulk paper. It was also found that the solder would not 'wick' onto the printed conductive surfaces under the action of flux. 

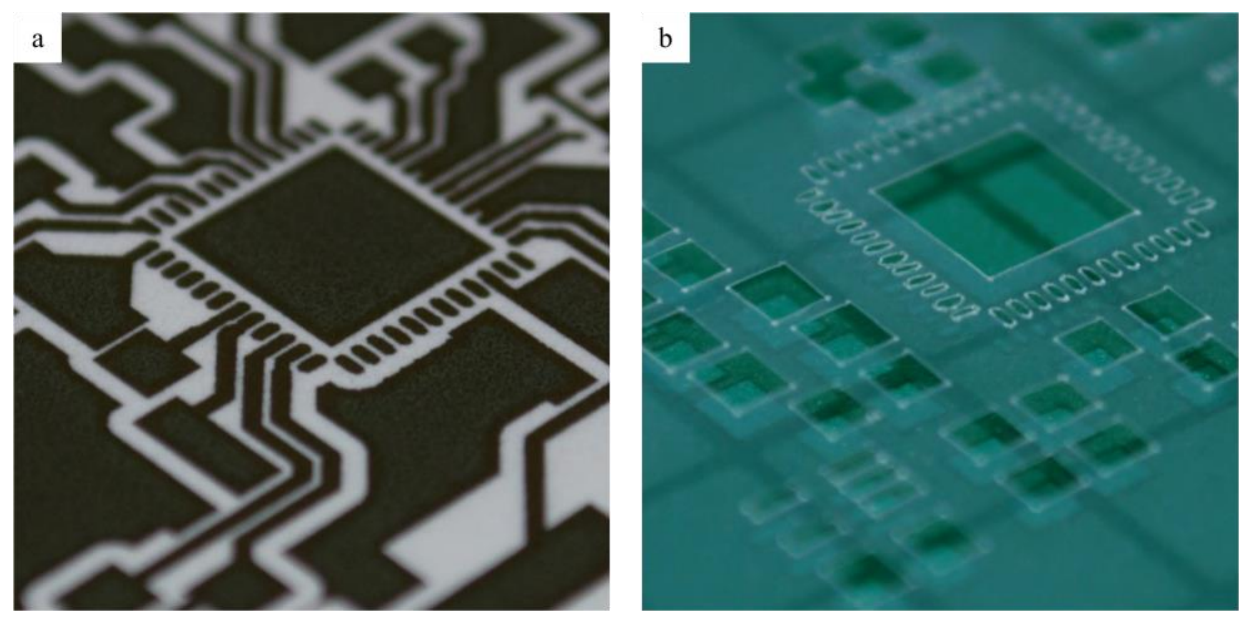

Figure 3. CIP PCB with complementary Mylar solder stencil. Despite being a standard consumer inkjet printer, the Brother MFC-J4410DW is easily able to produce CIP circuits at a resolution high enough to allow 48-pin QFN (Quad Flat No-leads package) chips (a). Similarly, the Silhouette Portrait craft paper cutter allows the rapid in-house production of high-resolution solder stencils for the application of conductive epoxy onto the CIP circuit.

A potentially exciting solution to the problem of temperature was outlined by Kawahara $e t a l,{ }^{8}$ via the use of electrically conductive transfer tape. This clear, thin tape, coated with adhesive on both sides, contains silver nano particles suspended in the adhesive. Such tapes are used to secure end connectors on flex PCBs, as the tape contains a concentration of nanoparticles that allows conduction through the tape, while avoiding lateral shorting between pins. This effect is enhanced by the application of pressure, which brings the particles into contact vertically.

We tested the transfer tape on CIP circuits. It was found that larger SMD components were able to receive signals and power through the tape without any applied pressure, and short circuits laterally did not occur. However, this effect was only demonstrated for SMD components of size 1206 and larger, presumably because the larger contact area of the pads on these components gave a much greater chance of a random conductive path forming through the tape. Furthermore, the effect was occasionally unreliable, and devices with smaller contact patches with the board e.g. TSSOP microcontrollers were only able to connect when considerable pressure was applied to the component. Although transfer tape is a very attractive solution for component connection and adhesion, it seems it is not an option for complex circuits with smaller components and connecting leads.

As a true low-temperature solution, a conductive epoxy has been used to construct the circuits found in this paper (CircuitWorks Conductive Epoxy, Onecall, UK). This two-part adhesive is normally used for the repair of conventional PCB circuits, but works well as an alternative to solder paste. The two parts are mixed 1:1, before being mixed for 100 seconds. We have found that the viscosity of the adhesive is critical for successful application, thus the exact mixing time. The epoxy is applied through an aligned solder stencil, before applying the components by hand under the microscope. One downside of the epoxy is that it cures at room temperature, with a potting time of around 10 minutes. This is in comparison to normal solder paste, which can often be left for months post application. However, 10 minutes is more than enough time to allow for the assembly of the circuits required for our application.

Room temperature curing would be ideal, but in reality results in lower than optimum adhesion and conductivity. As per the datasheet, the epoxy is cured on a hotplate (Quick 450-870ESD, KaiserTech, UK) at $70^{\circ} \mathrm{C}$ for 15 minutes. We have found that the photo paper does warp slightly at this temperature, but is not damaged. To further reduce warping, the CIP circuit is held down on a glass plate using low tack tape (e.g. masking tape), and this assembly is loaded onto the hotplate.

Solder stencils can be ordered from various companies, but this step could add several days to the turnaround time of a prototype, and lead to expense over time. We therefore aimed to produce solder stencils in-house. We 
originally used a laser cutter, using which we were able to produce accurate solder masks. Accuracy was improved by using the cutter's "raster" mode, which evaporates the material away, rather than cutting it out. This increases the accuracy of very small solder pad holes, as there is far less of a melt zone produced by burning through the edge of the holes in a single pass. We originally used standard OHP acetates (125 $\mu \mathrm{m}$ thickness) as the stencil material, but found that this deposited too much epoxy. We therefore moved on to $50 \mu \mathrm{m}$ thick Mylar sheeting, (Insulation Solutions, UK) which is strong and inexpensive (c. $£ 35$ for $90 \mathrm{~cm} \times 50 \mathrm{~m}$ ).

Even though the costs of laser cutters have dropped in recent years, the outlay for such machines is still in the tens of thousands of pounds, and is likely outside of the reach of many research groups and hobbyists. However, we have also tested a Silhouette Portrait (Silhouette America, USA) craft paper cutter, and found that it is an excellent alternative to laser cutting for use with CIP. The Portrait system operates like a combination of a laminator, an inkjet printer and a plotter system. The material to be cut is loaded onto a self-adhesive thin cutting mat, which is fed into the machine. The cut direction is controlled in the $\mathrm{Y}$-axis by the feed rollers, in the $\mathrm{X}$-axis by a horizontal mobile stage, and in the Z-axis (turning the cut off and on) by a spring loaded mechanism. The blade is mounted on a 360 bearing, which allows the blade to be dragged into the correct orientation as it is dragged across the paper.

The Silhouette Portrait cutter is more than capable of cutting solder stencils in Mylar for complex SMD circuits. In general, it has been found that the "tCream" layer exported from Eagle CAD into Corel Draw is slightly too big, and can often lead to shorting between components if they are placed too closely together. In a normal circuit with conventional solder paste, this would be mitigated by the way the paste wicks onto the conductive pads under the action of flux. For use with epoxy, we found reducing the size of the solder pads individually in Corel Draw by $10 \%$ prevented this issue.

Other components, such as TSSOP (Thin-Shrink Small Outline Package) microcontrollers, also require a reduction in the standard pad size in order to reliably prevent shorting between pins, or the weakening of the solder stencil by too many cuts being made in the same area. This is especially a problem when the 'overcut' mode is used, where the cutter can start a cut e.g. 100 um early in order to produce between quality 90 degree corners. We have found that reducing the pads for these devices laterally by $10-20 \%$ (i.e. to create more space between the pads) helps alleviate this issue.

When removed from the cutting mat, it is normally found that the cut material is still attached via a corner to the bulk material, even when overcut mode is used. In order to pull the cut material away, low tack tape can be applied to the bulk and pulled away. This sticks to and removes the cut material. This method can also be used to remove any cut material that has stuck to the cutting mat, as long as the tack of the tape is higher than that of the mat. It is therefore also recommended that the adhesive on the standard Silhouette mats is 'watered down' using a solvent e.g. isopropyl alcohol before use, as the standard adhesive is very strong. This can lead to damage to the Mylar on removal, and makes it nearly impossible to easily remove any cut material left behind.

The CIP circuits as describe above are complex, but they are only 'single sided'. Normal PCBs often contain at least two layers, which can be etched and connected by vias to form more complex and compact circuits. This is especially important in radio frequency (RF) circuits, as a solid ground plane is required. We have not yet found an easy way of creating true 'double sided' circuits, with components on both sides - the thin and flexible nature of the paper makes epoxy application to the backside of the paper difficult if components are already on the topside. This is compounded by a paucity of double-sided coated photo paper stock, and the inability of consumer printers to align prints accurately on both sides of the paper, either in two passes or via an automatic duplexer - alignment is generally only enough to make text look roughly in position, rather than accurate enough for circuit fabrication.

However, in this paper we demonstrate '1.5 sided' circuits. Our method uses the Silhouette Portrait to cut square via holes, $1 \mathrm{~mm}$ square, in the top layer of the circuit where ground, power or signal connections are required. This top layer is then aligned over a CIP bottom layer, and glued using a light-application spray glue onto the back side of the upper layer. The vias are included into the solder stencil design, increased in size by $20 \%$. This increase in size means that the epoxy is applied through the stencil in such a way that it overlaps the conductive area around the vias on the top CIP layer. Crucially, the via hole is big enough that the epoxy reliably sinks under its own weight into the hole, and makes a connection with the bottom layer. No further action is required to make the connection. The vias are small enough that they can be placed under the pads for both 1206 and 0805 components, giving direct grounding where desirable, and reducing the required board area. 

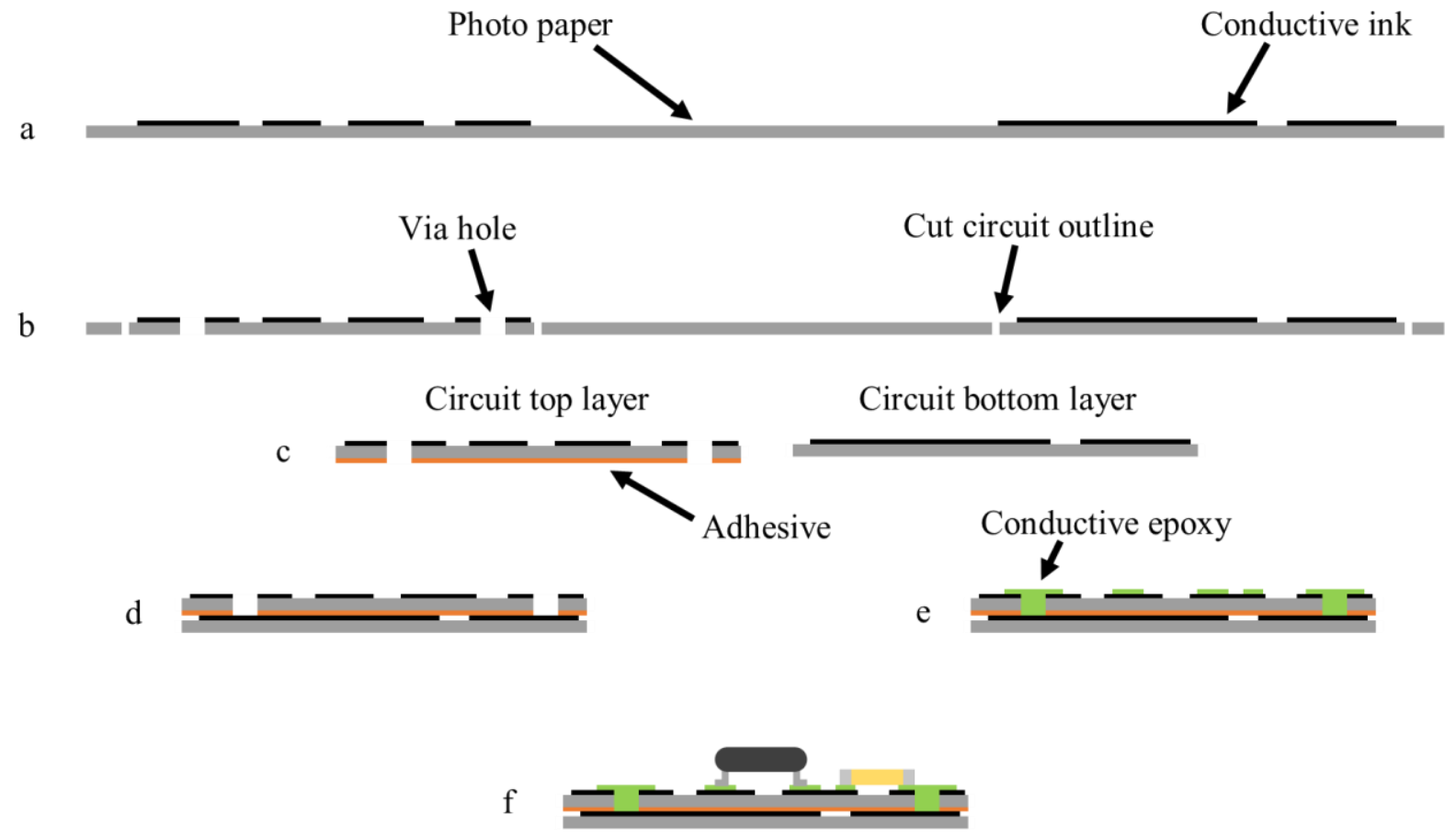

Figure 5. Complete process overview for production of 1.5-sided CIP circuits. The circuit design is printed onto the coated side of the photo paper, including the top (left) and bottom (right) layers of the circuit (a). The circuit is then cut away from the bulk of the paper, and via holes cut in the top layer, using a craft cutter $(b)$. The layers are then separated from the bulk, and the via holes cleared, before a light-application spray adhesive is applied to the back of the top layer (c). The two layers are aligned and bought into contact (d). Conductive epoxy is applied through a Mylar solder stencil, forming the component 'solder' pads and top-to-bottom layer vias (e). The required components are then placed manually onto the epoxy (f). Finally, the circuit is baked for 15 minutes at $70^{\circ} \mathrm{C}$ on a hotplate.

The cutting of holes in the upper CIP layer requires precise alignment of the cutter with respect to the existing printed circuit. Thankfully, the Silhouette Portrait has another useful feature - a photodiode that allows alignment of cuts to printed registration marks. As shown in Fig. 1, the registration marks are printed in the corners of the photo paper at the same time as the CIP circuit. This not only allows the cutting of the vias, but the edges of the layers of the circuit, and, as explored further in this paper, the outline of the eventual paper UAVs.

One unfortunate complication in the alignment process is that the photodiode cannot reliably see the registration marks when printed using the conductive ink on photo paper - it is assumed that there is insufficient contrast between the shiny surfaces of the printed ink and the photo paper, as normal ink, drying matte, is found correctly. To overcome this, we have cut stencils the same size as the alignment marks out of acetate sheets, which are the aligned over the printed marks and black ink applied through the stencil from a felt tip pen. It is hoped a more automated approach will be found in the near future. A graphical summary of the full process steps required to produce a 1.5 sided CIP circuit can be seen in Fig. 5.

\section{Circuit design and testing}

In order to produce our 'Papersonde', we decided on the following requirements. The sonde should be capable of measuring temperature, pressure, and potentially humidity. The data would be collected and processed by a microcontroller, with sufficient overhead for desired future features such as vehicle control. The board should contain at least a transmitter, but ideally a transceiver, capable of transmitting (and receiving) in the $433 \mathrm{MHz}$ UK amateur radio spectrum. Finally, it should contain a battery able to power the circuit for a number of hours. 


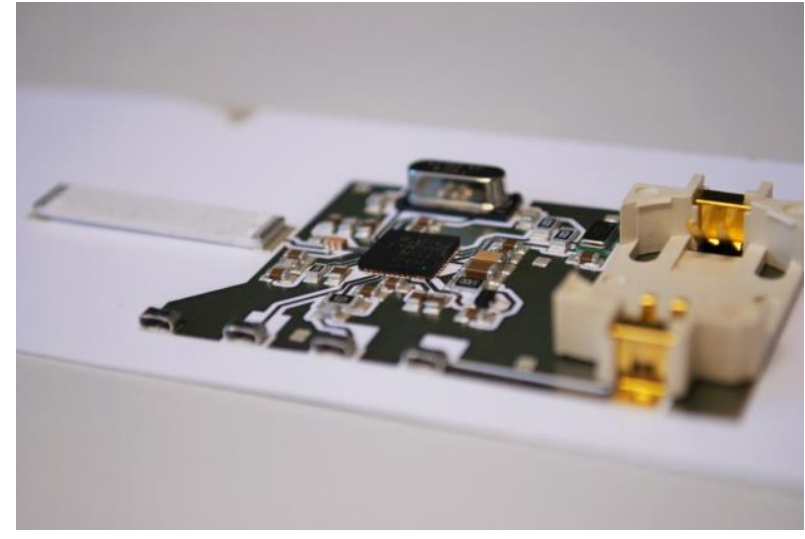

Figure 6. Finished CIP 'Papersonde'. This design includes a Texas Instruments CC430 QFN-48 microcontroller with integrated CC1101 sub-GHz transceiver (centre); a Measurement specialities barometer; a Microchip temperature sensor; and an SMD antenna (left) and CR2032 coin cell holder for mobile power (right).
The current prototype circuit is shown in Fig. 6. We chose a Texas Instruments (USA) CC430F5137IRGZT microcontroller as the heart of the papersonde system. This 16-bit chip features a maximum $20 \mathrm{MHz}$ clock speed, $4 \mathrm{kB}$ RAM and $32 \mathrm{kB}$ program memory, with 30 reconfigurable IO pins, and communication via I2C, SPI and UART with peripherals. However, its major advantage is its builtin sub-GHz $\mathrm{CC} 1101$ transceiver, which greatly reduces both circuit complexity and size. During the initial fabrication tests, it was found that the placing of the microcontroller was inhibited by the conductive epoxy patch deposited under the ground/thermal plane of the CC430 chip. This would often cause the chip to slip across the board on contact.

In the current prototype, a hole is cut through both layers of the board, and only the conductive epoxy required to connect the pins is deposited through the Mylar stencil. The circuit is then populated, and the epoxy cured. The board is then turned over, and further conductive epoxy applied manually through the hole directly onto the microcontroller ground plane, as well as on a number of top-to-back layer ground vias. The ground terminal of the battery is then adhered directly to the back of the board.

This design not only greatly eases the placement of the microcontroller, but we believe it will help with the thermal management of the electronics - the battery is only specified to function above $-20^{\circ} \mathrm{C}$ (higher than the anticipated required minimum temperature), and the microcontroller has been found to heat up significantly during prolonged transmission. It is therefore hoped that a direct electrical and thermal connection via the conductive epoxy will allow for better electrical grounding of the microcontroller; let the battery act as a heat sink for the CC430 chip; and conversely allow the microcontroller to act as a heater for the battery. This arrangement highlights the great flexibility of CIP technology, as this fully functional circuit was created from the previous design, which included a top-mounted battery holder, within a day of these problems being identified.

Pressure and temperature are provided by a Measurement Specialities (USA) MS5607-02BA03 SMD barometer. This low-power $(1 \mu \mathrm{A})$, low-cost sensor is simply integrated via an $\mathrm{I}^{2} \mathrm{C}$ bus to the microcontroller. A further temperature reading is provided by a Microchip MCP9700 thermistor, which outputs a temperature-dependent voltage that can be read by the microcontroller's ADC (analogue to digital convertor). Both components are able to output data down to $-40^{\circ} \mathrm{C}$, and the barometer can measure pressure down to $10 \mathrm{mbar}$ (c. $25,900 \mathrm{~m} / 85,000 \mathrm{ft}$. altitude). We have used an SMD antenna, tuned for $433 \mathrm{MHz}$ (Johanson Technology, CA, USA), and for testing an SMD battery holder for a CR2032 3V lithium battery. The current prototype circuit costs around $£ 15$ to assemble we hope this will drop as components are sourced in greater numbers.

The microcontroller is programmed over SPI-By-Wire using an MSP430 LaunchPad (Texas Instruments, USA) as the debugger. The program is written using open-source tools on Linux (Linux Mint 17), such as mspdebug, msp430-gcc, and standard text editing software e.g. nano, gedit. The SPI-By-Wire interface is accessed using a custom cable, connected via test probe pads on the CIP board.

Initial radio testing has been carried out in the laboratory. Various test signals have been transmitted and received locally using an Icom IC-R20 receiver (Icom Ltd., UK) with dl-fldigi HAB (high altitude ballooning) software. Promisingly, the signal was also picked up some $750 \mathrm{~m}$ away, through intervening buildings, by our receiving station at Highfield Campus, University of Southampton. This was unexpected, as the frequency and RTTY (radio teletype) standard we currently employ is generally limited to line-of-sign communication only. It is hoped that this success indicates that long-range clear line-of-sight communication will be easily possible, even 

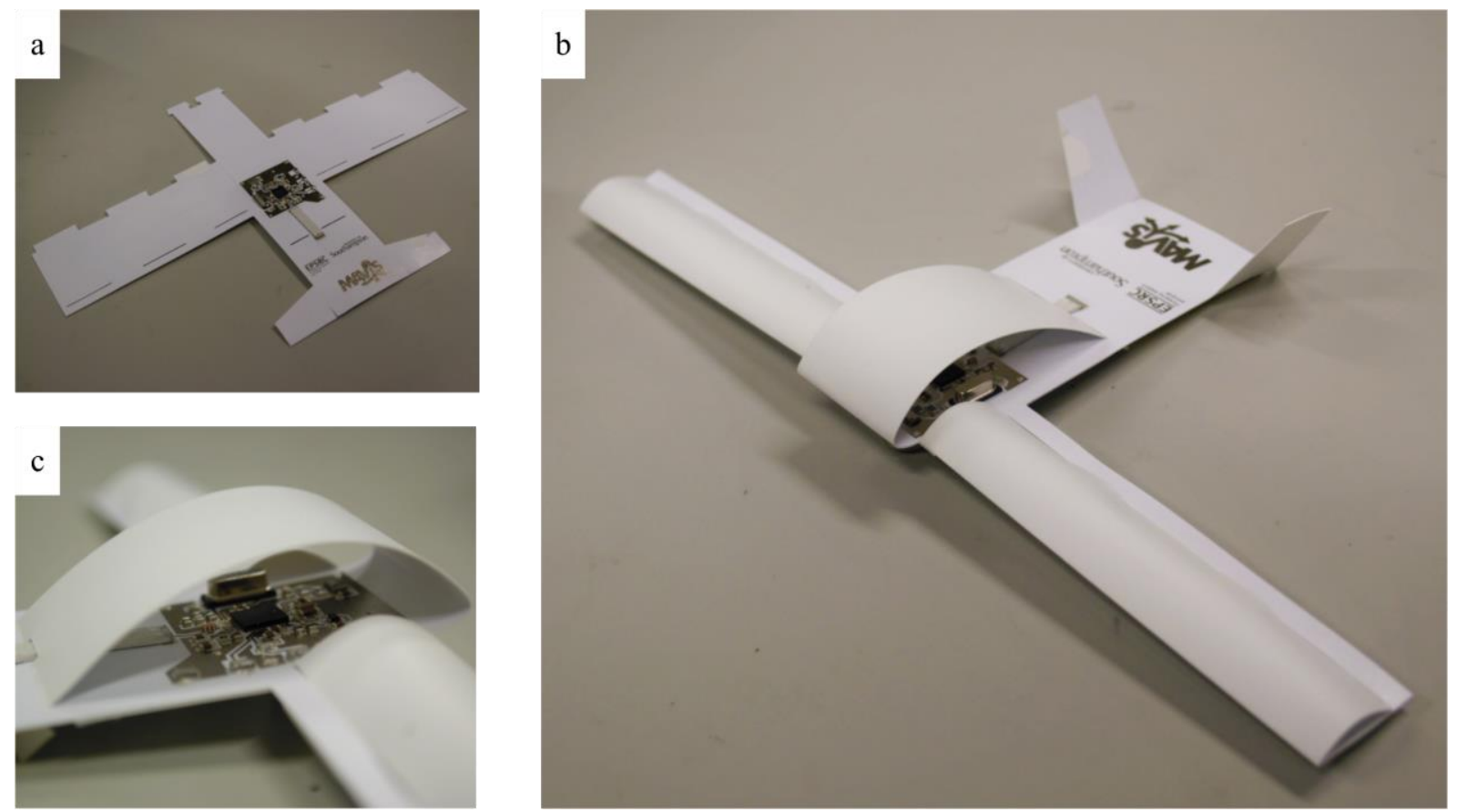

Figure 7. Prototype airframe with integrated 'Papersonde' circuit. CIP techniques on photo paper, along with the use of the craft paper cutter, allow the direct integration of paper circuits onto the airframe. As shown in (a), the cut airframe with integrated electronics, will remain flat until assembly is required at the time of the mission. This allows for ease of storage and transport, even shipping via conventional mail. We propose a design that is different from the traditional folded 'delta dart' paper aircraft, with a tab-and-slit assembly method, as shown assembled in (b). The airframe can be assembled quickly in few steps. The central fuselage has enough flat area to allow the direct printing of the circuit shown in Fig. 6, as shown in closeup in (c). In this design, as described in the text, the battery is attached directly to the bottom of the airframe, under the microcontroller..

using the SMD antenna.

The CC1101 transceiver built into the CC430 microcontroller does not support RTTY communication. As a result, we have used a 'bit-banging' technique, where the frequency of the CC1101's carrier signal is switched in software in order to encode data. This has successfully been used to transmit messages between paper circuits and our Icom receiver.

\section{Future Work}

The design and fabrication of potential airframes for the MAVIS fleet has been initiated, but requires much further work before a final design is settled upon. In any case, the flexibility, speed of turnaround and low-cost of CIP circuits means that any fleet could be comprised of a number of different models of micro UAV, and fleets could even be fabricated quickly via parametric design on a per-mission basis. A basic prototype plane can be seen in Fig. 7. The thickness of the photo paper allows for surprisingly stiff structures to be created, especially when the material is put under tension e.g. in the wings of the prototype UAV in Fig. 7b. The methods used to control the UAV are currently under investigation, and must also conform to the lightweight, low cost and low environmental impact parameters desired when designing the airframe. The MAVIS fleet will be programmed with pre-determined flightpaths, calculated in advance to best sample the desired volume of air. Work on this aspect of the system is ongoing. ${ }^{9}$

Future designs will be constrained in a number of ways. The circuit will be printed on the same substrate as the airframe, and the area of the circuit will need to be kept flat. Although the circuits are capable of withstanding some 
bending without damage, excessive bending can lead to the loss of components as the epoxy fails, or reduced conductivity in the ink. At the extreme end of this effect, it should be noted that the circuits cannot withstand folding, as the printed material cracks and flakes off the paper surface. This has led to a move away from the traditional 'delta dart' design of A4 paper airplanes, as this design greatly limits the position of sensors and actuators on the airframe.

It is also assumed that the aircraft will be cut from a single piece of paper. This allows completed circuits to potentially be sent out flat in the post in envelopes, to be assembled by researchers on site. The assembly will be via a tab-and-slit papercraft design, which gives increased reproducibility over folding. Where folds are required e.g. the front edge of the wing, the folds will be defined by serrated cuts through the paper, again giving better reproducibility. Finally, the design will incorporate some form of 'cockpit', which will protect the electronics, as well as providing shelter for the barometer, which should not be exposed to the elements directly, but, at the same time, must not be enclosed to an extent that would cause significant measurement lag at high rates of ascent or descent. The current design shown in Fig. 7 is simply a prototype airframe with the Papersonde circuit printed within the cockpit area. Future models will have a much more integrated design, with the electronics and airframe design process being as one, rather than two parallel processes brought together at an end point. It is hoped this synergy of design will allow for even more compact designs.

Further testing of the electronics will need to be carried out to assess their survivability in the field. It is hoped that the circuits will last long enough to complete their missions (around 6-12 hours) but as they are disposable, their functioning beyond this point is not required, nor desired - we expect it to degrade shortly after completing its mission. Initial balloon tests are planned for the near future.

\section{Conclusions}

CIP techniques have shown great promise for the production of nano-scale UAVs. The great flexibility of the technology, with every step being performed in-house, allows rapid turnaround of new designs, for both the electronics and the airframes. The total setup costs to start production are under $£ 1000$, and individual prototyping costs are also low. We have demonstrated a prototype 'Papersonde', along with an initial nano-UAV design, which will form the basis of the Massive Atmospheric Instrumentation System (MAVIS) currently under development

\section{References}

${ }^{1}$ Hunt, E. R., Jr., Hively, W. D., Fukikawa, S. J., Linden, D. S., Daughtry, C. S. T., and McCarty, Q. W., "Acquisition of NIR-Green-Blue Digital Photographs from Unmanned Aircraft for Crop Monitoring," Remote Sensing, Vol. 2, No. 1, 2010, pp. 290-305.

${ }^{2}$ Ramana, M. V., Ramanathan, V., Kim, D., Roberts, G. C., and Corrigan, C. E., “Albedo, atmospheric solar absorption and heating rate measurements with stacked UAVs", Quarterly Journal of the Royal Meteorological Society, Vol. 133, 2007, pp. 1913-1931.

${ }^{3}$ Zang, W., Lin, J, Wang, Y., and Tao, H., "Investigating small-scale water pollution with UAV Remote Sensing Technology", World Automation Congress (WAC) Conference, IEEE, Puerto Vallara, Mexico, 2012, pp. 1-4.

${ }^{4}$ Pounds, P. E. I. and Singh, S. P. N., "Integrated electro-aeromechanical structures for low-cost, self-deploying environment sensors and disposable UAVs", 2013 IEEE International Conference on Robotics and Automation (ICRA), Karlsruhe, Germany, (4444-4451). 6-10 May 2013.

${ }^{5}$ van Osch, T. H. J., Perelaer, J., de Laat, A. W. M., and Schubert, U. S., "Inkjet Printing of Narrow Conductive Tracks on Untreated Polymeric Substrates”, Advanced Materials, Vol. 20, No. 2, 2008, pp. 343-345.

${ }^{6}$ Rida, A., Yang, L., Vyas, R. and Tentzeris, M. M., "Conductive Inkjet-Printed Antennas on Flexible Low-Cost Paper-Based Substrates for RFID and WSN Applications”, IEEE Antennas and Propagation Magazine, Vol. 51, No. 3, pp 13-23.

${ }^{7}$ Yoshiki, T., Shino, S. and Kobayashi, K., Mitsubishi Paper Mills Limited, Tokyo, Japan, U.S. Patent for "Process for Preparing Conductive Material" \#8,012,676 B2, 6 ${ }^{\text {th }}$ September 2011.

${ }^{8}$ Kawahara, Y., Hodges, S., Cook, B. S., Zhang, C. and Abowd, G.D., "Instant Inkjet Circuits: Lab-based Inkjet Printing to Support Rapid Prototyping of UbiComp Devices", UbiComp '13, ACM International Conference on Ubiquitous Computing, September 2013

${ }^{9}$ Crispin, C. and Sobester, A., “An Intelligent, Heuristic Path Planner for Multiple Agent Unmanned Air Systems”, AIAA SciTech 2015, AIAA Infotech @ Aerospace, Kissimmee, Fl, USA, January 2015. 